\section{Just a minute}

\author{
Stephen Hancocks OBE \\ Editor-in-Chief
}

The popular and long running BBC Radio 4 entertainment programme Just A Minute famously asks its contestants to speak for sixty seconds on a given subject without hesitation, repetition or deviation. It sounds as if it is a simple enough task but the fun of the challenge and the difficulty of achieving it attests to the programme's appeal and longevity. However, it is a discipline that we might rehearse ourselves since the ability to communicate succinctly and accurately with our patients is a skill well worth cultivating.

Two recent circumstances have brought this need back into sharp relief, each featured in this issue; the now growingly infamous HTM 01-05 on decontamination (page 100) and antibiotic prophylaxis in relation to infective endocarditis (page 114 and online article E5). In the former the need is to communicate why we are being forced to implement procedures to a much greater extent than has ever been deemed necessary previously; while in the latter, thanks to the evidence-based consensus of the National Institute of Health and Clinical Excellence (NICE), the requirement is to explain why it is now no longer necessary to take a hitherto required action.

In both circumstances the important factor which will enable us to continue to provide the most appropriate care for our patients is the trust that they have in us as competent clinicians and as trained professionals with their best interests at heart. In the case of a patient who has previously received antibiotic prophylaxis as a prelude to dental treatment, the alarm of being told that this is no longer judged as being needed is understandable. In such situations, calm and clear explanation is the only ethical course, even though the temptation might be to take the line of least resistance and merely prescribe the antibiotic anyway, for a quiet life. Ultimately it will be the trust, or otherwise, that a patient has for their dentist which will make the difference.

\section{PATIENTS' CONTINUED TRUST}

With regard to decontamination that trust is implicit by virtue of the fact that patients continue to attend for examinations and treatment. If they did not feel that they were entering a safe environment they would not turn up unless perhaps under the duress of toothache or the lack of an alternative. We are all familiar with patients questioning us over various things that appear in the media from time to time and which trigger concerns or doubts. It might be about tooth whitening, lasers, oral cancer and may also include cross-infection control. Years ago there were worries, for example, over the spread of HIV if the practice was known to also treat HIV positive patients, but in the intervening time and in the absence of any evidence whatsoever of patients being at such cross-infection risk in the dental setting, it is no longer a rational concern.

One suspects that, by extension, the same is true of other infectious conditions. The media would be quick to magnify the risks of going to the dentist if cases of MRSA, for instance, were to have been traced to dental practices or even if anecdotal suspicion was raised through clusters of infective cases. To date, despite the thousands, millions of courses of treatment undertaken every day, every year in the UK and elsewhere, no such data exist.

That is not to say that patients are unlikely to raise the matter. Indeed it provides us with an opportunity to use our decontamination measures as indicators of our commitment to care for our patients. There is an assumption that we do but there is no harm in making it clear. It is akin to bakers announcing in their windows that their sandwiches are freshly made that day. We would assume that they are but the statement of fact reinforces the message and encourages confidence.

One other factor is also valuable to keep in mind in this communication, namely that ultimately it is the patient who shoulders the cost of all such safeguards, whether through government raised finance or directly by private contract. So, if, for instance certain cross-infection control measures were deemed to be in excess of what was actually necessary then it would be the patient who paid for such inverse cost-benefit.

It is a difficult concept to grasp, just as the reversal of the antibiotic practice takes explanation and requires understanding. There is always a danger too that emotion overtakes logic and expediency; that political convenience suffocates rational pragmatism. Which is where one might reasonably expect a National Institute to be able to play a useful role. If it can judge matters which pertain to individual life and death situations why not allow it to examine the circumstances around other proposals that potentially affect us all? In talking to our patients we might break the rules of Just A Minute because we will need to repeat the messages, to hesitate is human and to deviate is a casualty caused by dealing with the questions raised by the individuals that we treat. But there is another twist here because the ever resourceful English language allows us a further interpretation that would not be lost on the contestants; that of asking for further consideration. The regulations say what? Come on, just a minute...

DOI: 10.1038/sj.bdj.2010.108 